\title{
LOCALLY FINITE RING VARIETIES
}

\author{
AWAD A. ISKANDER
}

\begin{abstract}
Necessary and sufficient conditions are given for a variety of associative rings to be locally finite. These conditions are utilized to show that a variety is generated by a finite ring if, and only if, it contains only finitely many subvarieties. Also, the Everett extension of a variety by another variety is a locally finite variety (a variety generated by a finite ring) if, and only if, each of the varieties is locally finite (generated by a finite ring).
\end{abstract}

All rings considered here are associative and not necessarily with 1. $A$ variety (an equational class) of algebras is a class of algebras closed under homomorphic images, subalgebras and Cartesian products; or equivalently, it is the class of all algebras satisfying a set of identities (cf., e.g., [1], [2], [3], [9], [10], [11]). A variety is said to be locally finite if every finitely generated member is finite. We show that a locally finite ring variety is precisely a variety satisfying $m x=0$ and $x^{r}+x^{r+1} q(x)=0$ for some positive integers $m, r$ and some $q(x) \in \mathbf{Z}[x]$. In [6], R. L. Kruse shows that the identities of a finite ring are finitely based; the arithmetical ring case is shown by H. Werner and R. Wille [13]. From [6], it also follows that a finite ring generates a variety containing only finitely many subvarieties. We show here that the converse is also true. If $\mathfrak{U}$ and $\mathfrak{B}$ are ring varieties, then the class $\mathfrak{U} . \mathfrak{B}$ of all rings possessing an ideal belonging to $\mathfrak{U}$ whose factor belongs to $B$ is a variety [4], [8], [9], [10]. We show that the set of all locally finite varieties (varieties generated by a finite ring) is closed under products and hence under lattice joins and meets.

1. In a locally finite variety, every member generated by one element is finite. There are varieties of groups whose cyclic members are finite, but the varieties are not locally finite: the Burnside varieties of groups, $x^{n}=1$, $n$ is sufficiently large [10]. The situation is different for rings.

Presented to the Society, January 23, 1975; received by the editors March 21, 1974.

AMS (MOS) subject classifications (1970). Primary 08A15, 16A38; Secondary 16A06, 16A44.

Key words and phrases. Locally finite varieties of associative rings, equational classes, lattice of varieties, polynomial identities, free members in a variety, variety generated by a finite ring. 
Theorem 1. For a variety $\mathfrak{B}$ of rings, the following conditions are equivalent:

(1) $\mathfrak{B}$ is locally finite.

(2) Every ring in $\mathfrak{B}$ generated by one element is finite.

(3) For some positive integers $m, n$ and for some polynomial $q(x) \in \mathbf{Z}[x]$ of degree $<n-1, \mathfrak{B}$ satisfies $m x=0, x^{n}+x q(x)=0$.

(4) For some positive integers $m, r$ and for some polynomial $t(x) \in \mathbf{Z}[x]$, $B$ satisfies $m x=0, x^{r}+x^{r+1} t(x)=0$.

Proof. All the conditions are satisfied in the variety of one element rings. We can assume that $\mathfrak{B}$ contains a ring with a nonzero element. It is clear that $(1) \Rightarrow(2)$. Assume (2). Hence $F_{1}(\mathfrak{B})$-the free ring of $\mathfrak{B}$ on one generator-is finite of characteristic $m>1$. Let $m=p_{1}^{a_{1}} \cdots p_{s}^{a_{s}}$ be the prime factorization of $m$. If $\mathbb{B}_{i}$ is the subvariety of $\mathfrak{B}$ satisfying $p_{i}^{a_{i x}=0,1 \leq i}$ $\leq s$, then $B=B_{1} \times \cdots \times B_{s}$. So, it will be sufficient to check the case of varieties $\mathfrak{B}$ satisfying $p^{k} x=0$, where $p$ is a prime. Consider the subvariety $\mathfrak{B}^{\prime}$ of $\mathfrak{B}$ satisfying $p x=0 . F_{1}\left(\mathfrak{B}^{\prime}\right) \cong x \mathbf{Z}_{p}[x] / I$, where $I$ is a $T$-ideal of $x \mathbf{Z}_{p}[x] . \quad I$ is principal, i.e., $I=x g(x) \mathbf{Z}_{p}[x]$ for some $g(x) \in \mathbf{Z}_{p}[x]$. As $F_{1}\left(\mathbb{B}^{\prime}\right)$ $\in \mathfrak{B}, F_{1}\left(\mathbb{B}^{\prime}\right)$ is finite and $g(x) \neq 0$. Also, as $\mathfrak{B}$ contains a ring of more than one element, $g(x) \notin \mathbf{Z}_{p}$. Thus $x g(x)$ is of degree $\geq 2$. Thus $\mathfrak{B}^{\prime}$ satisfies $x g(x)=0$, i.e., $\mathfrak{B}^{\prime}$ satisfies $x^{n}+x q(x)=0$ and $x^{r}+x^{r+1} b(x)=0$ for $n=$ degree of $x g(x)$ and $r=$ order of $x g(x)$, degree of $q(x)<n-1, n>1, r \geq 1$. But $F_{1}\left(\mathfrak{B}^{\prime}\right) \cong F_{1}(\mathfrak{B}) / p F_{1}(\mathfrak{B})$, and $p^{k-1}\left(p F_{1}(\mathfrak{B})\right)=0,\left(p F_{1}(\mathfrak{B})\right)^{k}=0$. If $a \epsilon$

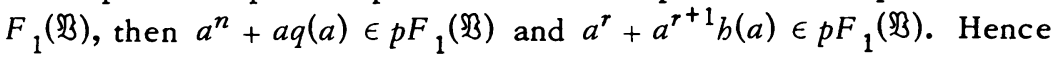

$$
\left(a^{n}+a q(a)\right)^{k}=0=\left(a^{r}+a^{r+1} b(a)\right)^{k},
$$

i.e., $B$ satisfies conditions (3) and (4).

Conditions (3) and (4) are equivalent for rings of prime characteristic. Assume (3). If $A \in \mathfrak{B}$, then $A / p A \in \mathbb{B}^{\prime}$ and $B=A / p A$ is an algebra over the prime field $\mathbf{Z}_{p}$. If $A$ is finitely generated, so is $B$. Hence by a result of I. Kaplansky [5] (in case $A$ is finitely generated) $B$ is finite dimensional over $\mathrm{Z}_{p}$, i.e., $B$ is finite. By a result of $\mathrm{J}$. Lewin [7], $p A$ is a ring of finite index in $A$; and $p A$ is finitely generated. Also $p A$ is nilpotent of characteristic $p^{k-1}$; therefore, $p A$ is finite. Thus $|A|=|p A| \cdot|B|$ is finite, concluding the proof of Theorem 1 .

Corollary 1. For any ring varieties $\mathfrak{U}$ and $\mathfrak{B}, \mathfrak{U} \cdot \mathfrak{B}$ is locally finite if, and only if, $\mathfrak{U}$ and $\mathbb{B}$ are locally finite. 
Proof. $\mathfrak{U}, \mathfrak{B} \leq \mathfrak{U} \cdot \mathfrak{B}$. If $\mathfrak{U} \cdot \mathfrak{B}$ is locally finite, $\mathfrak{U}$ and $\mathfrak{B}$ are subvarieties of a locally finite variety, and hence are locally finite. Conversely, if $\mathfrak{U}$ and $\mathfrak{B}$ are locally finite, then $\mathfrak{U}$ satisfies $m x=0, x^{r}+x^{r+1} q(x)=0$; and $\mathfrak{B}$ satisfies $n x=0, x^{s}+x^{s+1} t(x)=0$, for some $m, n, r, s>0$ and $q(x), t(x) \epsilon$ $\mathbb{Z}[x] . \mathfrak{U} \cdot \mathfrak{B}$ satisfies

$$
m n x=0=\left(x^{s}+x^{s+1} t(x)\right)^{r}+\left(x^{s}+x^{s+1} t(x)\right)^{r+1} q\left(\left(x^{s}+x^{s+1} t(x)\right)\right) .
$$

2. The variety of all rings satisfying $p x=0, x^{p}=0(p>1)$ has infinitely many subvarieties. However, a finite ring generates a variety with only finitely many subvarieties [6]. In fact, the converse is also true.

Theorem 2. A ring variety contains only finitely many subvarieties if, and only if, it is generated by a finite ring.

Proof. Let $\mathfrak{B}$ be a variety possessing only finitely many subvarieties Denote by $\mathfrak{B}_{n}$, the subvariety of $\mathfrak{B}$ generated by a free member of $\mathfrak{B}$ of rank $n, n=1,2,3, \cdots$. Then $\mathfrak{B}_{1} \leq \mathfrak{B}_{2} \leq \cdots \leq \mathfrak{B}_{n} \leq \cdots \leq \mathfrak{B}$, and $\mathfrak{B}$ is the lattice join of all $\mathfrak{B}_{n}[2],[9],[10]$. Since $\mathfrak{B}$ contains only finitely many subvarieties, this chain must be stationary, i.e., there is $k>0$ such that $\mathbb{B}_{n}=\mathbb{B}_{k}$ for all $n>k$, i.e., $\mathfrak{B}=\mathfrak{B}_{k}$. That is, $\mathbb{B}$ is generated by a finitely generated member. By Theorem 1 , it will be sufficient to show that $B$ satisfies condition (3). First $F_{1}(\mathfrak{B})$ is of positive characteristic; otherwise, $F_{1}(\mathfrak{B}) / p F_{1}(\mathbb{B})$ $\neq 0$ for every prime $p$, and $B$ would contain an infinite family of minimal varieties [12]. Thus $F_{1}(\mathfrak{B})$ is of characteristic $m>0$. We can assume that $m>1$. If $m=p_{1}^{a_{1}} \cdots p_{s}^{a_{s}}$ is the prime factorization of $m, \mathfrak{B}=\mathfrak{U}_{1} \times \mathfrak{U}_{2} \times \cdots \times$ $\mathfrak{U}_{s}$ where $\mathfrak{U}_{i}$ is the subvariety of $\mathfrak{B}$ of all rings satisfying $p_{i}^{a_{i x}=0,1 \leq i}$ $\leq s$. Thus, it will be sufficient to check the case $\mathbb{B}$ satisfies $p^{n} x=0 ; p$ is a prime. Let $\mathfrak{B}^{\prime}$ be the subvariety of $\mathfrak{B}$ of all rings satisfying $p x=0$. Then

$$
F_{1}\left(\Re^{\prime}\right) \cong x \mathbf{Z}_{p}[x] / x g(x) \mathbf{Z}_{p}[x]
$$

If $g(x)=0$, then $F_{1}\left(\mathfrak{B}^{\prime}\right) \cong x \mathbf{Z}_{p}[x]$ and $\mathfrak{B}^{\prime}$ contains all commutative rings of characteristic $p$ and hence an infinite number of subvarieties. Hence $g(x) \neq$ 0 , i.e., $B^{\prime}$ satisfies $x^{r}+x^{r+1} q(x)=0, p x=0$, where $r-1$ is the order of $g(x)$, from which, we infer that $\mathfrak{B}$ satisfies condition (3).

Theorem 3. For any ring varieties $\mathfrak{U}$ and $\mathfrak{B}, \mathfrak{U} \cdot \mathfrak{B}$ is generated by $a$ finite ring $i f$, and only if, each of $\mathfrak{U}$ and $\mathfrak{B}$ is generated by a finite ring.

Proof. If $\mathfrak{U} \cdot \mathfrak{B}$ is generated by a finite ring, $\mathfrak{U} \cdot \mathfrak{B}$ contains only finitely many subvarieties. Thus $\mathfrak{U}, \mathfrak{B} \leq \mathfrak{U} \cdot \mathfrak{B}$ contain only finitely many subvarieties. Hence by Theorem 2 , each of $\mathfrak{U}$ and $\mathfrak{B}$ is generated by a finite ring. 
Denote by $\mathfrak{S}(m, n, e)[6]$, where $m, n, e$ are positive integers, the class of all rings $A$ with the property $m A=0$, every primitive homomorphic image of a subring of $A$ is a simple finite ring of order dividing $n$, and if $B$ is a nilpoient homomorphic image of a subring of $A$, then $B^{e}=0$. It was shown in [6, Theorem 8.1$]$ that $\mathfrak{S}(m, n, e)$ is a variety generated by a finite ring. Noticing that every finite ring belongs to some $\mathfrak{S}(m, n, e)$, Theorem 3 will be proved if we show

Claim. $\mathfrak{S}(m, n, e) \cdot \mathfrak{S}(k, l, f) \leq \mathfrak{S}(m k,[n, l]$, ef $)$, where $[n, l]$ is the least common multiple of $n, l$.

Since $\mathfrak{S}(m, n, e) \cdot \mathfrak{S}(k, l, f)=\Omega . \Omega$ is a variety, it will be sufficient to show that if $A \in \Omega \cdot \Omega$, then $k m A=0$; if $A$ is nilpotent, then $A^{e f}=0$; and if $A$ is primitive, then $A$ belongs to $\Omega$ or $A$ belongs to $\Omega$.

Indeed $A \in \Omega \cdot \Omega$ iff there is an ideal $B$ of $A$ such that $B \in \Omega$ and $A / B$ $\epsilon$ ?. So $m B=0$ and $k(A / B)=0$, i.e., $k A \leq B$, and hence $m k A \leq m B=0$. If $A$ is primitive, it also satisfies $x^{r}+x^{r+1} q(x)=0$ for some $r>0, q(x) \in \mathbf{Z}[x]$ (since $\Omega$ and $\Omega$ are locally finite and so is $\Omega \cdot \Omega$ ). By Kaplansky's result [5], $A$ is simple. So, either $B=A$, i.e., $A \in \Omega$, or $B=0$, i.e., $A \in \Omega$. If $A$ is nilpotent, both $B$ and $A / B$ are nilpotent. Hence $B^{e}=0$ and $(A / B)^{f}=0$, i.e., $A^{f} \subseteq B$ and so

$$
A^{e f}=\left(A^{f}\right)^{e} \subseteq B^{e}=0
$$

3. From Corollary 1, we conclude that the set of all locally finite ring varieties is an ideal of the lattice of all ring varieties. It is closed under variety product. The set of varieties that are not locally finite is an ideal of the multiplicative groupoid of ring varieties. The set of all varieties each of which is generated by a finite ring is an ideal of the lattice of all ring varieties strictly contained in the ideal of locally finite varieties. By Theorem 3, it is closed under variety product; the set of varieties not generated by any finite ring is an ideal of the multiplicative groupoid of ring varieties.

The author thanks the referee for a number of valuable remarks.

\section{REFERENCES}

1. G. Birkhoff, Lattice theory, 3rd ed., Amer. Math. Soc. Colloq. Publ., vol. 25, Amer. Math. Soc., Providence, R. I., 1967. MR 37 \#2638.

2. P. M. Cohn, Universal algebra, Harper \& Row, New York, 1965. MR 31 \#224. $\# 1320$.

3. G. Grätzer, Universal algebra, Van Nostrand, Princeton, N. J., 1968. MR 40

4. A. A. Iskander, Product of ring varieties and attainability, Trans. Amer. Math. Soc. 193 (1974), 231-238.

5. I. Kaplansky, Topological representation of algebras. II, Trans. Amer. Math. Soc. 68 (1950), 62-75. MR 11, 317. 

318.

6. R. L. Kruse, Identities satisfied by a finite ring, J. Algebra 26 (1973), 298-

7. J. Lewin, Subrings of finite index in finitely generated rings, J. Algebra 5 (1967), 84-88. MR 34 \#196.

8. A. I. Mal'cev, Multiplication of classes of algebraic systems, Sibirsk. Mat. Ž. 8 (1967), 346-365 = Siberian Math. J. 8 (1967), 245-267. MR 35 \#4140.

9. - Algebraic systems, "Nauka", Moscow, 1970; English transl., Die Grundlehren der math. Wissenschaften, Band 192, Springer-Verlag, Berlin and New York, 1973. MR 44 \#142.

10. Hanna Neumann, Varieties of groups, Ergebnisse der Mathematik und ihrer Grenzgebiete, Band 37, Springer-Verlag, New York, 1967. MR 35 \#6734.

11. J. M. Osborn, Varieties of algebras, Advances in Math. 8 (1972), 163-369. MR $44 \# 6775$.

12. A. Tarski, Equationally complete rings and relation algebras, Nederl. Akad Wetensch. Proc. Ser. A 59 = Indag. Math. 18 (1956), 39-46. MR 18, 636.

13. H. Werner and R. Wille, Charakterisierungen der primitiven Klassen arithmetischer Ringe, Math. Z. 115 (1970), 197-200. MR 42 \#318.

DEPARTMENT OF MATHEMATICS, UNIVERSITY OF SOUTHWESTERN LOUISIANA, LAFAYETTE, LOUISIANA 70501 